\title{
Hemorrhagic Stroke: A Diagnosis Guided by Visual Field Findings
}

\author{
Nicole Riese, OD, FAAO \\ Staff Optometrist, \\ VA New Jersey Health \\ Care System
}

\author{
Melissa Bailey, MD \\ University of Miami School \\ of Medicine
}

This material is the result of work supported with resources and the use of facilities at VA New Jersey Health Care System, Michael E. DeBakey Veterans Administration Medical Center, and the Katy Veterans Administration Outpatient Clinic. The contents do not represent the views of the U. S. Department of Veterans Affairs or the United States Government.

\begin{abstract}
This case report outlines a patient with hemorrhagic stroke who presented to the eye clinic with visual field loss, which the patient interpreted as blur, and a severe headache. The visual field loss consisted of an incomplete homonymous hemianopsia with an absolute superior defect, which corresponded well to the right-sided temporal lobe hemorrhage found on head computed tomography (CT) scan. This case highlights the importance of all eye care providers recognizing stroke symptoms and understanding referral timelines and protocols as well as the high predictive value of visual fields.
\end{abstract}

\section{KEY WORDS:}

Hemorrhagic stroke, homonymous hemianopsia, headache, arteriovenous malformation

\section{INTRODUCTION}

Stroke trails only ischemic heart disease as the most common cause of death worldwide; in 2010, it is estimated that 5.9 million people died from stroke. ${ }^{1}$ Stroke is defined as either an infarction or hemorrhage in the brain, retina, or spinal cord that causes a sudden-onset focal neurologic defect lasting longer than 24 hours or when imaging shows an infarct or hemorrhage corresponding to symptoms of any duration. ${ }^{1}$ Symptoms of a stroke vary, but commonly include unilateral weakness, numbness, visual changes including diplopia, altered speech, ataxia, and non-orthostatic vertigo. ${ }^{1}$ A recent study found that new visual changes attributed to stroke are present in $48 \%$ of patients when admitted for stroke and in $60 \%$ of stroke survivors; visual changes include impaired central vision in $56 \%$, eye movement abnormalities in $40 \%$, visual field loss in $28 \%$, visual inattention in $27 \%$, and visual perceptual disorders in $5 \% .^{2}$

Accurately identifying a patient undergoing an acute stroke can at times be challenging, especially if symptoms are atypical or varying, or if imaging is either not available or if the results appear normal. Visual field loss in particular can be a powerful tool that can provide useful information for predicting where pathology may exist. An infarct or hemorrhage posterior to the optic nerve chiasm will cause bilateral visual field loss with respect to the vertical midline. A lesion causing a complete homonymous hemianopsia cannot be further localized other than that it is located posterior to the chiasm on the contralateral side of the visual field loss. Incomplete hemianopsias can provide more information; in brief, more congruous defects tend to be more posterior on the visual pathway ${ }^{3}$. Additionally, a superior visual field defect tends to result from damage to the temporal lobe, and inferior visual field defects tend to result from damage to the parietal lobe. ${ }^{3}$

\section{CASE REPORT}

A 52-year-old male presented to the eye clinic due to mild blurred vision in both eyes and a severe headache. Five days prior to presentation, the patient was watching television when he had a sudden onset of a throbbing, left-sided headache which he rated subjectively as 10/10 pain, associated with photophobia. Upon awakening the next morning, he noted what he described as blurry vision in both eyes. He had experienced a similar episode in the 1990s which was diagnosed as a migraine. On ex- 
amination, the patient saw 20/20 with the right eye, 20/25 with the left eye, and a superior left quadrantanopsia was suspected from confrontation fields using the National Institutes of Health Stroke Scale (NIHSS) technique. ${ }^{4}$ The rest of his dilated exam was similar to his full exam one month prior without relevant findings. A formal visual field was performed using the Humphrey Field Analyzer (HFA) with the 24-2 SITA Standard testing strategy (Carl Zeiss Meditec, Dublin, CA), which confirmed an incomplete left homonymous hemianopsia, with an absolute superior left quadrantanopsia (Figure 1). A lesion in the patient's right temporal lobe was suspected, and the patient was sent to the Emergency Department (ED) with a copy of the exam findings.

Figure 1: (a,b) Visual fields performed on the day of initial presentation. An incomplete left homonymous hemianopsia, with an absolute superior left quadrantanopsia, is apparent. A neurologic etiology was the most likely cause of these visual field defects.

a)

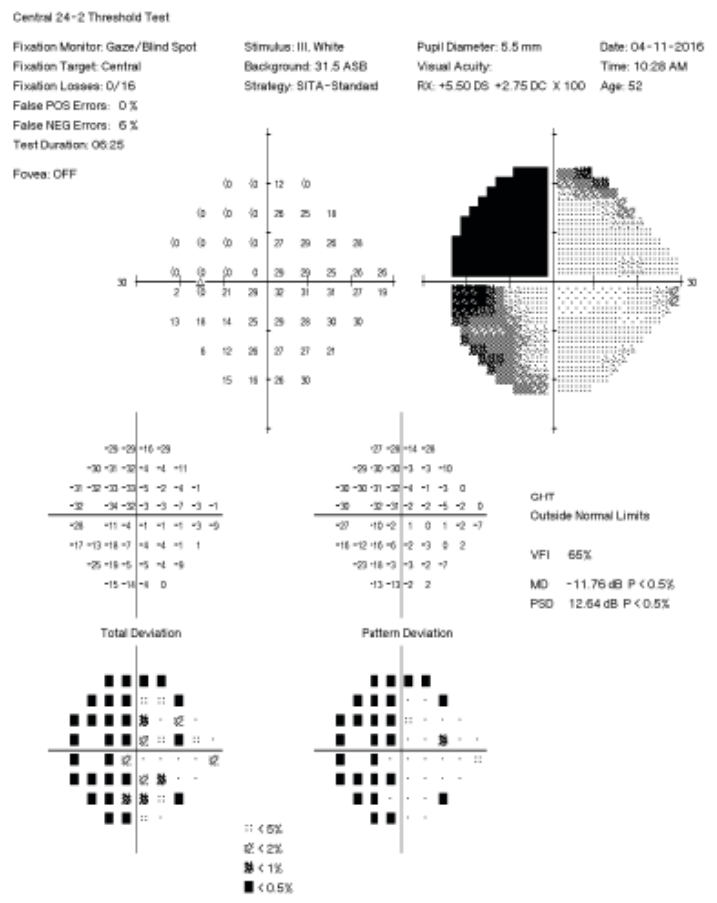

b)

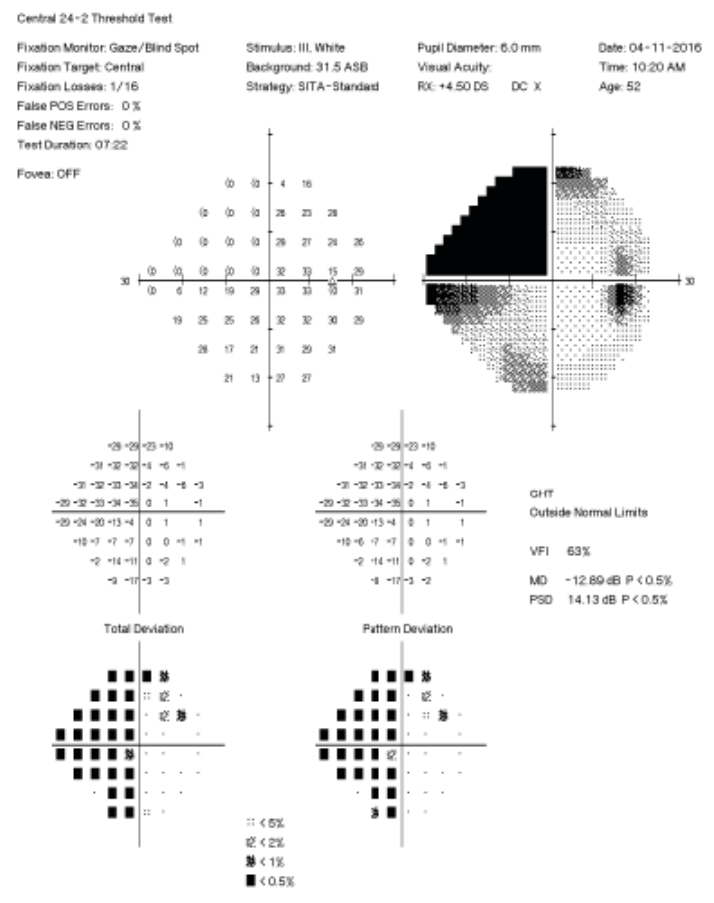

At the ED, a head computed tomography (CT) scan revealed a large right temporal parenchymal hemorrhage, a specific type of intracranial hemorrhage (ICH), with an associated $4 \mathrm{~mm}$ leftward midline shift (Figures 2,3). The lesion was measured as $6.1 \times 3.3 \times 1.8 \mathrm{~cm}$ in size. The patient was admitted to the Medical Intensive Care Unit and monitored closely. A CT angiogram and magnetic resonance imaging (MRI) scan with and without contrast were performed, but did not reveal the source of the bleed. His full inpatient work-up also did not reveal the etiology of the ICH, although arteriovenous malformation (AVM) was the suspected etiology given his young age, appearance and location of the bleed, and lack of other medical conditions. After discharge, his subsequent outpatient scans showed resolution of the ICH about four months later.

Approximately one year from the initial presentation, a cerebral angiogram revealed a right temporal Martin-Spetzler grade $1 \mathrm{AVM}$ as the source of the $\mathrm{ICH}$, meaning the patient had an abnormal connection between an artery and a vein that bypassed the capillary bed and created an area that was prone to bleeding. Grade 1 AVMs are small and have the lowest surgical risk; this patient desired surgical correction of the AVM to help prevent the possibility of a future bleed and a successful operation was performed the next month. The patient continues to be followed at the low-vision clinic and repeat visual fields have shown a stable, absolute superior left quadrantanopsia, with improve- 
ment in the inferior quadrant. As the visual field defect persisted longer than 24 hours and was directly caused by the $\mathrm{ICH}$, this would be classified as a hemorrhagic stroke. He has stopped driving due to his field loss and continues to note difficulty seeing objects on his left side, more difficulty reading, and trouble with his balance. He is still working on prism training and with various rehabilitation therapists.

Figure 2: Axial view of the diffusion-weighted MRI. The hypointense, or dark, region on the left side of the image is the patient's right temporal lobe and represents the area of hemorrhage.

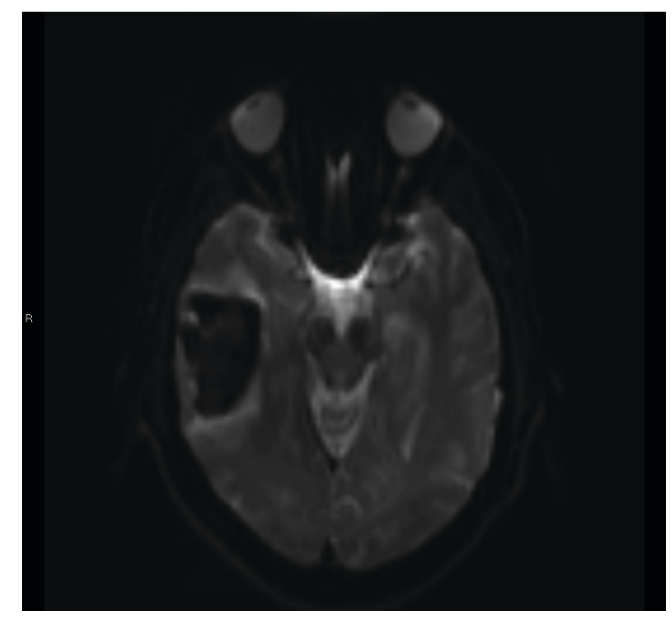

Figure 3: Sagittal view of the T1-weighted MRI. The area of hyperintensity, or bright area, shows the location and size of the temporal lobe hemorrhage.

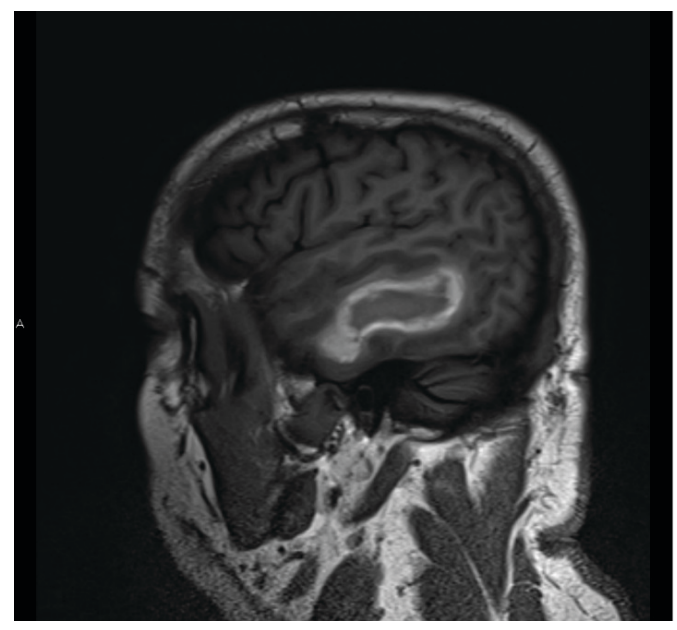

\section{DISCUSSION}

In this case, the congruity of the visual field defects with respect to the vertical midline strongly suggested a neurologic etiology. Additionally, a full ocular exam ruled out any retinal or optic nerve disorders that would account for the visual field findings. Therefore, the main differential diagnoses remaining before the referral to the ED would include migraine with ophthalmic manifestations, longstanding visual field defect from prior stroke or neurologic event, or a new mass or other lesion or infarct likely affecting the right temporal lobe. Although the patient had a history of migraines, and it is possible that he had a congruous defect, ${ }^{3}$ this is rare, and more serious underlying etiologies needed to be ruled out. It was unlikely that this visual field defect was longstanding from a prior stroke or neurologic event 
given that the patient noted new ocular symptoms. Based on the appearance of the visual field defects and the associated headache, the most likely differential was new infarct or hemorrhage affecting the right temporal lobe, and thus urgent same-day referral was indicated. The patient's headache was especially concerning for cerebral hemorrhage given its characteristics. A headache associated with intracranial hemorrhage often shows a sudden onset and may be classified as a "thunderclap headache", which is a severe headache that reaches maximum intensity within one minute. ${ }^{5}$ The headache is typically located on the same side of the head as the hemorrhage, although in this case it was on the opposite side. Other concerning features would be nausea and vomiting, alteration in level of consciousness, neck stiffness, seizure, severe hypertension, or associated neurological deficits such as unilateral facial droop or weakness; this patient did have an associated neurological deficit of visual field loss. ${ }^{6}$

A diagnosis of intracranial hemorrhage comes with an estimated one-month mortality rate of 35-50\%, with a poorer prognosis for larger hemorrhages, decreased level of consciousness on admission, and midline shift amongst other findings.$^{78}$ Importantly, while ICH can be attributed to a variety of underlying etiologies, when ICH is due to an AVM rupture, the outcome is typically more favorable. ${ }^{9}$ Patients with AVM-associated ICH tend to be significantly younger with fewer known disease and stroke risk factors. However, even after controlling for these variables, these patients have a lower risk of death and higher rates of favorable discharge. ${ }^{9}$

While this patient's AVM was not diagnosed until after the ICH had cleared and cerebral angiography was performed, other patients may either have known preexisting conditions or will have abnormalities that are discovered during initial imaging. When a patient is hospitalized for an ICH due to AVM, the standard practice in some hospitals is to recommend surgical resection during the initial hospitalization..$^{10}$ A typical AVM has an estimated annual rupture rate of between 1-4\%. ${ }^{9}$ The results of the ARUBA study (A Randomized Trial of Unruptured Brain Arteriovenous Malformations) suggest that unruptured AVMs should be managed conservatively, with medical rather than surgical management. ${ }^{10}$ However, in this case, due to the prior AVM rupture, the patient elected to have the AVM surgically resected.

As demonstrated in this case, quality of life is often affected after homonymous visual field loss. In some jurisdictions, patients with homonymous hemianopsias are no longer able to drive due to visual field requirements, and in regions that do not have visual field requirements for driving, it has been shown that such patients have reduced ability to detect pedestrians or respond to unexpected events. ${ }^{3}$ Numerous factors related to field loss contribute to a reduction in reading speed and comprehension in many patients. ${ }^{3}$ Many patients with homonymous hemianopsias may benefit from further referrals, especially to low-vision for prism therapy, driving rehabilitation, or occupational therapy. ${ }^{3}$ The patient should also be educated that, in most cases of homonymous hemianopsia, the visual field defects may improve over the course of a month or two, but are unlikely to improve after six months unless an underlying cause is corrected. ${ }^{3}$

Without the visual field findings in this patient, it is likely the ICH would not have been diagnosed in a timely manner. This case highlights the importance of performing an urgent visual field for cases of atypical or new headaches and especially for cases where the patient may present to an optometrist prior to seeing a primary care physician. In this specific case, the field loss was significant enough to be detected before the automated fields were performed, but in other cases an automated field may be the only way to detect field loss. When a patient is symptomatic and once visual field loss indicating a neurologic etiology is discovered, the appropriate referral is to the emergency department for immediate testing and management as the patient may have a life-threatening condition. •

REFERENCES

1. Hankey GJ. Stroke. Lancet. 2017; 389: 641-654. DOI: 10.1016/S01406736(16)30962-X.

2. Rowe FJ, Hepworth LR, Howard C, Hanna KL, Cheyne CP, Currie J. High incidence and prevalence of visual problems after acute stroke: An epidemiology study with implications for service delivery. PLOS ONE 2019; 14(3): 1-16. DOI: 10.1371/journal.pone.0213035

3. Goodwin D. Homonymous hemianopia: challenges and solutions. Clin Ophthalmol. 2014; 8: 1919-1927. DOI: 10.2147/OPTH.S59452

4. National Institute of Neurological Disorders and Stroke. NIH Stroke Scale. https:/www.ninds.nih.gov/sites/default/files/NIH_ Stroke_Scale_Booklet.pdf. Accessed August 26, 2019.

5. Ducros A, Bousser MG. Thunderclap headache. BMJ. 2013; 346e: 8557. DOI: 10.1136/bmj.e8557

6. An SJ, Kim TJ, Yoon BW. Epidemiology, risk factors, and clinical features of intracerebral hemorrhage: an update. J Stroke. 2017; 19(1): 3-10. DOI: 10.5853/jos.2016.00864
7. Suthar NN, Patel KL, Saparia C, Parikh AP. Study of clinical and radiological profile and outcome in patients of intracranial hemorrhage. Ann Afr Med. 2016; 15: 69-77. DOI: 10.4103/1596-3519.176259

8. Morotti A, Goldstein JN. Diagnosis and management of acute intracerebral hemorrhage. Emerg Med Clin North Am. 2016; 34(4): 883-899. DOI: 10.1016/j.emc.2016.06.010

9. Murthy SB, Omran SS, Gialdini G, et al. Outcomes after intracerebral hemorrhage from arteriovenous malformations. Neurology. 2017; 88: 1882-1888. DOI: 10.1212/WNL.0000000000003935

10. Mohr JP, Parides MK, Stapf C, et al. Medical management with or without interventional therapy for unruptured brain arteriovenous malformations (ARUBA): a multicenter, non-blinded, randomised trial. Lancet. 2014;383(9917):614-621. DOI: 10.1016/S01406736(13)62302-8 